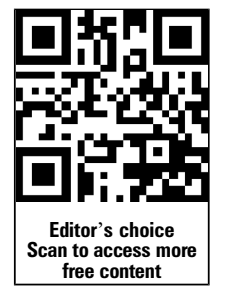

- Additional material is published online only. To view please visit the journal online (http://dx.doi.org/10.1136/ heartjnl-2013-304852).

${ }^{1}$ Faculty of Nursing, University of Alberta, Edmonton, Canada ${ }^{2}$ School of Nursing, McMaster University, Hamilton, Canada ${ }^{3}$ Faculty of Health Sciences, Australian Catholic University, Melbourne, Australia ${ }^{4}$ School of Health, Glasgow Caledonian University, Glasgow, Scotland

\section{Correspondence to} Professor Alexander M Clark, Faculty of Nursing, University of Alberta, 11405-87 Avenue, Edmonton, Alberta, Canada T6G 1C9;

alex.clark@ualberta.ca

Received 26 August 2013 Revised 7 January 2014 Accepted 17 January 2014 Published Online First 18 February 2014
To cite: Clark AM Spaling $\mathrm{M}$, Harkness $\mathrm{K}$ et al. Heart 2014:100 716-721.

\title{
Determinants of effective heart failure self-care: a systematic review of patients' and caregivers' perceptions
}

\author{
Alexander M Clark, ${ }^{1}$ Melisa Spaling, ${ }^{1}$ Karen Harkness, ${ }^{2}$ Judith Spiers, ${ }^{1}$ \\ Patricia H Strachan, ${ }^{2}$ David R Thompson, ${ }^{3}$ Kay Currie ${ }^{4}$
}

\section{ABSTRACT}

Context Disease management interventions for heart failure (HF) are inconsistent and very seldom incorporate the views and needs of patients and their caregivers into intervention design.

Objective and data To improve intervention effectiveness and consistency, a systematic review identified 49 studies which examined the views and needs of patients with HF and their caregivers about the nature and determinants of effective HF self-care.

Results The findings identify key drivers of effective self-care, such as the capacity of patients to successfully integrate self-care practices with their preferred normal daily life patterns and recognise and respond to HF symptoms in a timely manner.

Conclusions Future interventions for HF self-care must involve family members throughout the intervention and harness patients' normal daily routines.

\section{BACKGROUND}

Heart failure (HF) self-care matters, but what matters most in HF self-care? Reducing the immense and growing burden of HF depends as much or more on promoting effective self-care and disease management as on medicines. ${ }^{1-3}$ Yet, attaining proficiency in HF self-care is difficult-it involves patients becoming adept in a very wide range of skills across multiple domains on a daily basis. ${ }^{1} 2$ These include monitoring weight objectively every day, adhering to a complex medication regimen, ensuring fluid restriction and management, and engaging in behaviours related to fluid intake, diet, physical activity, symptom monitoring and help-seeking. ${ }^{1} 245$ Interventions to promote effective HF self-care have been somewhat successful but are often inconsistent. ${ }^{6-14}$ Beyond the wide scope and complexities of HF self-care, this is likely because HF self-care interventions are themselves complex, diverse, and often vaguely described, ${ }^{15}$ have different effects in different populations ${ }^{16}$ and may be confounded in trials with pharmacological regimen.

More effort needs to be devoted to understanding HF self-care prior to interventions being evaluated via randomised trials. ${ }^{17}$ Indeed, the Medical Research Council Framework for the Design and Evaluation of Complex Interventions suggests that 'user perspectives' are valuable and vital to incorporate into intervention design before formal evaluation via trials. ${ }^{18}$ The rationale for this recommendation is to increase the responsiveness of the intervention to the actual needs as opposed to the anticipated needs of the patient and thereby improve the overall effectiveness of the intervention.

Yet, there is evidence that HF self-care interventions are seldom based on data relating to patients' perceptions of their own needs. ${ }^{19}$ Families, particularly spouses/partners, though frequently involved in daily self-care, have been consistently excluded from almost all past HF interventions. ${ }^{17} \quad 1920$ Knowledge of patients and caregivers has then rarely been incorporated into the design and content of interventions evaluated in past studies. ${ }^{17}$ To inform the design of future interventions and promote effective HF self-care, a systematic review of qualitative studies was performed to examine patients' and caregivers' needs, experiences and preferences regarding the nature and determinants of HF self-care.

\section{METHODS}

As this study was focused on the complex factors and processes that influence self-care rather than preidentified predictors of self-care, qualitative research studies were the focus of this review. Qualitative systematic review has been used to understand various aspects of health around disease management ${ }^{21} 22$ and, importantly, is not dependent on using studies that self-identify (eg, via titles and abstracts) as being related only to 'self-care'. This is vital when reviewing qualitative research of $\mathrm{HF}$ because studies are often framed in general terms (eg, 'patient experiences') but may contain themes and data relating to HF 'self-care'. As both patients and caregivers are involved in self-care and supported by healthcare professionals, studies including and pertaining to each were included. HF self-care was defined in the review as 'the decisions and strategies undertaken by the individual in order to maintain life, healthy functioning and well being. ${ }^{23}$ To support consistent interpretation between the research team, data or themes were interpreted to be relevant if: findings related to any process, phenomena, or construct that pertains to the self-care of HF in patients or support of self-care by lay caregivers.

\section{Study selection}

To be included in this review, studies had to report primary qualitative data wholly or as part of mixed methods design, contain population-specific data or themes from adults (18 or older) reasonably seen to pertain to HF self-care, and be published as full papers/theses during or after 1995. The search 
strategy combined general and specific terms relating to HF and qualitative design and was used to search the following databases until 19 March 2012: Ovid MEDLINE, Ovid EMBASE, Ovid PsycINFO, CSA Sociological Abstracts, Ovid AARP Ageline, EBSCO Academic Search Complete, EBSCO CINAHL, EBSCO SocINDEX, ISI Web of Science and Scopus. We also searched Proquest Dissertations and Theses database, scanned reference lists of recent papers, and consulted with colleagues. Only English papers were included due to lack of resources for translation. All papers identified by the systematic search were screened for relevancy first by their titles/abstract. Papers which appeared to be potentially relevant were then full-text screened against the inclusion criteria.

Meta-synthesis ${ }^{24}$ was used to provide a new account of the phenomena being explored and involved studies were reanalysed and compared with each other to produce new theories or knowledge. A four-stage approach was used to synthesise the data. ${ }^{24}$ First, studies were read fully and data/themes extracted verbatim in relation to findings pertaining to the nature or determinants of HF self-care across all domains of HF self-care. Data from the studies were extracted into the Joanna Briggs Institute software for systematic review (Qualitative Appraisal and Review Instrument) and then analysed for common themes taking account of the nature and quality of the component studies. This approach has been described more fully in past reviews to understand cardiac patient perspectives, including previous reviews of people with heart disease ${ }^{25}$ and $\mathrm{HF}^{26}$

The quality of all included studies was assessed using the criteria from the Critical Appraisal Skills Programme (CASP) Qualitative Appraisal Tool. ${ }^{27}$ Studies were ranked, based on the CASP Tool criteria, as low, moderate or high quality, but were not excluded on the basis of quality. Both screening and quality appraisal involved independent assessment by two reviewers and disagreements were resolved by discussion among the research team. Multiple publications of the same study populations were included as independent studies as the publications contained varying themes and findings related to the self-care of HF; however, the descriptions of the study populations for these papers were only included once.

\section{RESULTS}

\section{Search results}

Of 1421 papers identified (figure 1), 63 papers met the criteria for inclusion in the meta-synthesis. A subset of these themes, representing 49 studies, is presented in this review of self-care needs (see online supplementary table S1). Studies involved: 1446 patients (214 sex not reported; 596 women; 636 men; mean age 65.6, range 25-98 years), 186 caregivers and 63 health professionals. With some exceptions, populations were predominantly Caucasian and urban dwelling. Over half the studies were conducted in the USA $(n=28)$. Overall, the study quality was moderate $(n=27)$ with common study weaknesses being superficial analyses of themes, over-reliance on convenience sampling and insufficient description of sample characteristics (see online supplementary table S1).

\section{Review findings}

Key limitation affecting self-care: basic HF self-care knowledge deficits still exist

Achieving effective HF self-care was widely seen to be very challenging to patients mostly due to difficulties remembering: what self-care behaviours were important ${ }^{28-30}$ or appropriate to $\mathrm{do}^{28}{ }^{31}$ and the harmful effects ${ }^{32}$ and uncontrollability of symptoms. ${ }^{31} 3334$
Though studies were from different countries and time periods, the studies consistently indicated the wide prevalence of fundamental gaps in patient and caregiver knowledge and understanding about HF self-care. Participants demonstrated or reported a low knowledge of $\mathrm{HF}^{35-38}$ or lack of understanding of self-care ${ }^{36-41}$ by:

- Lack of recall about basic elements of the nature of $\mathrm{HF}^{36} 3842-44$

- Apparent misattribution of HF symptoms to other conditions, ${ }^{3745}$ age $^{46}$ or medications ${ }^{47}$

- Low understanding of the links between signs ${ }^{44}$ or symptoms of HF or the heart ${ }^{33} 383948-50$

- Absence of references to the importance of weight management or monitoring ${ }^{37}$

- Avoidance ${ }^{51}$ or low awareness ${ }^{39}$ of the severity of HF.

Some studies specifically identified that patients' capacity to engage in effective HF self-care was reduced by low knowledge generally 3352 or in relation to particular domains of self-care, including:

- Sodium reduction 37384453

- Medication adherence 37444850

- Weight monitoring 445253

- Physical activity. ${ }^{4154}$

A lack of knowledge was perceived to contribute to confusion, ${ }^{35}$ delays in help-seeking, ${ }^{39} 49$ uncertainty over future illness trajectory ${ }^{35}$ and how to self-care. ${ }^{35} 49$ There was little evidence from the studies that patients recognised these challenges existed. General misconceptions and low knowledge of HF symptoms were seen to contribute to ineffective self-care. ${ }^{54}$ For example, some patients did not understand that sodium is salt, and despite reporting limited intake of salt, failed to restrict sodium. ${ }^{53} 55$ Consequently, health professionals could view patients as non-adherent even though patients reported practices they believed to align with recommendations. ${ }^{55}$

Key contextual factor affecting self-care: HF self-care is shared Informal or lay caregivers, usually spouses/partners, were very frequently cited as contributing daily and positively to patients' HF self-care. ${ }^{19} 28 \quad 34364244475456-63$ Caregiver support was seen to facilitate adherence, ${ }^{34}$ especially during times of symptom exacerbation (when adherence may be most critical), ${ }^{58}$ when caregivers could play a more prominent role in self-care. $^{58}$

Caregiver actions around self-care often responded to patient wellness and capacities. Hence, caregivers' contributions ranged widely from promoting patient independence to assisting with basic needs. ${ }^{19} 61$ Irrespective of patient wellness, caregivers reported constant, yet 'invisible' observation and assessment of the patient. ${ }^{19}$ Over time, caregivers could develop a repertoire of subtle cues that signalled fluctuations in patients' conditions; for example, changes in pallor or mood reflected alterations in patient wellness, which the patient ${ }^{56}$ or providers did not seem to recognise. ${ }^{19}$ Although the types of support provided by caregivers ranged substantially, they most frequently facilitated two aspects of self-care:

- Medication management ${ }^{19} 4754$ 60-62 6465

- Sodium reduction, including grocery shopping and meal preparation. $^{28} 42475457$

Key skill 1: integrating self-care with normal life patterns

Patients and caregivers reported a number of key higher-level skills and abilities that promoted effective self-care. These included the ability to integrate self-care recommendations with everyday social and occupational routines 444952 and therefore 

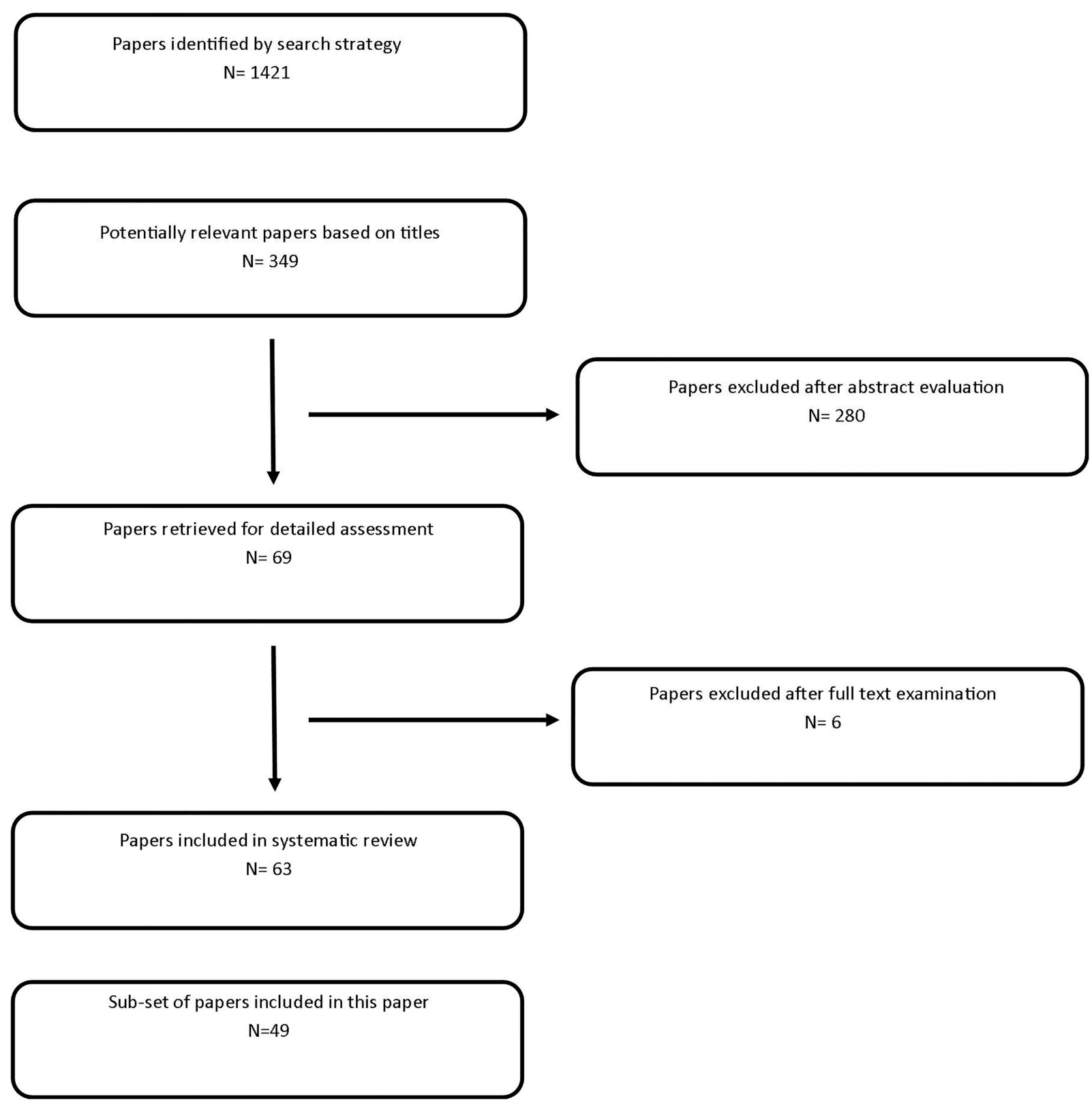

Figure 1 Flow of studies from identification to inclusion.

live a more 'normal' life. ${ }^{66}$ Integrating HF self-care strategies into everyday life routines was especially effective in increasing reported adherence. ${ }^{44}{ }^{45}$ Cues commonly arose in the context of daily life ${ }^{60}$ 67-69 that facilitated successful 'juggling' of both self-care and daily activities. ${ }^{42} 69$ These cues included visual reminders (eg, locating medicines on the breakfast table or television), using meals and morning routines to structure medication doses, ${ }^{67}$ and verbal reminders from caregivers. ${ }^{42}{ }^{67}$ Patients also reported altering medication dosages (in accordance with overall prescriptions) when needed in response to symptom changes. ${ }^{39444958676970}$ Devices to organise medications were cited as being helpful by some patients, including: pill boxes, ${ }^{44} 57677172$ blister packs ${ }^{48}$ and advance preparation of medicines. $^{71}$

Due to reliance on these clues, alterations to established daily life routines could also reduce reported adherence to medicines regimen. ${ }^{52} 5369$ For example, shifting occupational and social demands could impede successful management of diuretics. $^{49}$
Key skill 2: timely symptom detection, recognition and action A key HF self-care skill was the early detection of subtle changes in symptoms. ${ }^{4653} 54$ The ability to learn about self-care over time through formative experiences ${ }^{49}$ was important as patients developed links between self-care and knowledge of HF to form a complex but coherent framework for effective management. ${ }^{38} 68$

Patients who downplayed the severity of their symptoms tended to delay longer in seeking help ${ }^{39} 70$ and misattribute symptoms to age ${ }^{3946}$ or comorbidities. ${ }^{48}$ Patients also reported an over-reliance on subjective symptoms, ${ }^{54} 73$ fear of healthcare, ${ }^{73} 74$ past negative experiences and symptom ambiguity, ${ }^{38} 54$ which can reasonably be seen to contribute to delayed helpseeking. To judge the severity of $\mathrm{HF}$, subjective indicators were relied upon, such as feeling nervous or faint, ${ }^{54}$ although this is disagreed upon elsewhere in patients who participated in HF specialist clinics and reported keeping consistent records of weight and oedema assessment, and other symptom management strategies. $^{71}$ 
Key skill 3: caregivers, their knowledge and the range of HF self-care

Though caregivers provided substantial support, as noted above, for medication adherence and sodium avoidance, very few studies cited caregiver assistance with other aspects of self-care as recommended by clinical guidelines, such as daily weighing, ${ }^{44}$ fluid restriction, ${ }^{44}$ physical activity ${ }^{42}$ or timely help-seeking. ${ }^{19}{ }^{63}$ These facets of self-care were also not commonly cited by patients. Of the 49 included studies, there were no reports of caregivers promoting and/or supporting smoking cessation or reduced alcohol intake for HF patients.

In the same way that formal knowledge could influence patient capacity to manage HF, lack of knowledge about HF could limit the extent to which caregivers could provide clinically effective care. ${ }^{19}{ }^{72}$ Although caregivers saw their role as indisputable and rewarding, ${ }^{61}{ }^{64}$ they could also feel socially isolated, distressed, ${ }^{61}{ }^{64}$ fatigued $^{51}$ and ill-prepared ${ }^{64}$ for the demands of caregiving. Indeed, patients and caregivers cited a need for greater informational support for caregivers. ${ }^{51} 636475$ For example, although caregivers in one study reported monitoring patients for peripheral oedema, the same caregivers did not report monitoring fluids or sodium intake or indicate knowledge of the links among these. ${ }^{19}$ This suggests that while caregivers appear to be motivated, willing and involved daily in supporting the patient at home, the care provided may not always contribute positively to management. However, no studies reported that caregivers detracted from management or worsened HF symptoms.

\section{Key skill 4: caregivers fostering patient independence}

While poor support at home could lead to difficulty with managing self-care, ${ }^{36}$ lack of support was not limited simply to patients who lived alone. For example, some caregivers were cognisant of the potential to over-care. ${ }^{19}$ Indeed, one study cited a patient's complete dependence on her partner, despite her capacity to manage some aspects of self-care independently. ${ }^{50}$

\section{DISCUSSION}

HF self-care is key to improving outcomes and reducing HF disease burden. The findings from this review indicate that despite more focus on HF self-care in guidelines and interventions, gaps in key aspects of patient and caregiver knowledge and skills remain across studies irrespective of time, study or country of setting. Despite these basic gaps, there was also widespread evidence that patients were keen to manage their HF and used creative and collaborative approaches to daily management -trying to integrate self-care with their normal routines, working extensively with family caregivers, seeking to detect and respond to early symptoms, and fostering independence. While both patients and caregivers in the studies appeared willing to uptake self-care recommendations, they often did not have the basis to do so effectively. In other instances, domains of self-care were more notable by their absence-for example, around the importance of smoking cessation, alcohol restriction and weight and fluid management. Instances in which life patterns changed or demands fluctuated also appeared to have negative implications for adherence.

These trends raise questions in the light of past trials. It is surprising that basic knowledge deficits remain despite these being readily amenable to being addressed via normal clinical care and/or disease management programme interventions. That said, knowledge is not synonymous with skill-and the relationship(s) between HF knowledge and skills around self-care appear to be non-linear. For example, other factors, such as cognitive effects of $\mathrm{HF}^{76}$ or poor mental health, ${ }^{77}$ may account for this in patients but are unlikely to influence caregivers. More understanding is needed regarding the moderating influence of these facets. That basic aspects and practices of HF self-care recommended by guidelines, such as objective weight monitoring, were seldom evident in the study findings is significant but cannot itself be taken as strong evidence that HF self-care behaviours were not being undertaken or were seen as unimportant. Absence of evidence, famously, is not necessarily evidence of absence. Yet, the qualitative data suggest that patients and, often, caregivers did not see these aspects as being important or influential in terms of health.

Based on the findings of this review, care and interventions to facilitate effective HF self-care should draw on clinical guidelines relating to the main domains of HF self-care, but also:

1. Include both patients and their nominated caregivers in clinical consultations; scope exists to provide useful support in primary, community and hospital settings.

2. Seek first to understand patient and caregiver beliefs about $\mathrm{HF}$ and its self-care, their expectations and aspirations for daily life, for example, via use of open-ended questions or structured rapid patient surveys. General skills around HF self-care can be assessed objectively and quickly ${ }^{23}$ and basic knowledge should not be assumed.

3. Address self-care beliefs held by patients and caregivers that are contrary to best research evidence, or do not promote acceptance and assimilation of diagnosis, sensitively with research-informed alternatives. Common barriers to understanding and comprehension (such as low cognitive functioning) can be assessed reliably and rapidly in clinical care using validated measurement tools. ${ }^{78}$

4. Integrate patient values, expectations and aspirations with clinical guideline recommendations and adapt care recommendations to ensure congruence accordingly.

5. Foster daily reliance on objective measures of symptoms and encourage urgency in responding to identified worsening symptoms.

6. Harness cues in patients' home environments and routines to increase compliance and adopt a problem-solving approach that involves developing contingencies when life patterns are altered.

7. Recommend pill boxes to all patients to manage medicines prior to and after hospital discharge; pharmacists can provide specialist advice to ensure technologies are appropriate to particular patients. Address the purposes of medications with all patients and their caregivers.

These steps are likely to improve the effectiveness and consistency of future disease management interventions to promote HF self-care. In relation to other facets of design, there appear to be few patient or caregiver-related barriers to harnessing the contributions of health professionals from various disciplines, different sectors or with technology. This raises the issue of whether future care strategies should focus on discrete 'disease management programmes' (usually provided by specialists) that complement the rest of the patient's healthcare or support that is more integrated with the rest of patient healthcare.

While there is likely to be more specialist expertise in HF and its self-care in acute hospital settings, this specialist care is also potentially less sustainable and accessible: health professionals in primary care and family medical settings and community pharmacists all stand as potentially positive and influential sources of support for HF self-care. Yet, the contributions of these various groups should be weighed against the importance of ensuring care 
is coordinated, accessible, and reduces regimen complexity and treatment burden. ${ }^{79}$ The use of patient checklists, decision-aids and electronic health records offers promise in supporting better and more integrated care across multiple care providers. However, the effectiveness of each of these tools in isolation to improve care continuity or outcomes in complex chronic diseases has yet to be shown. ${ }^{80-82}$ Current evidence therefore supports continued evolution of discrete disease management interventions to supplement care. Attempts should be focused on improving the design of the components of these disease management programmes using the recommendations of this review.

In addition, more knowledge is needed of HF self-care. Survey research is needed that systematically evaluates the knowledge and problem-solving abilities of patients and caregivers around each main domain of HF self-care to measure: knowledge, problem-solving, understanding, confidence and reported behaviours. In addition to examining the influence of sex, age and symptom severity, international comparisons should be made across patients in countries with different health systems and wealth levels to understand whether macro systems characteristics influence these individual-level factors.

Technology offers promising ways to integrate personalised support from health professionals and technology. For example, basic knowledge of HF and self-care could be raised and reinforced and symptoms monitored using social media via cell phone or tablet technology to provide ongoing reminders about key aspects of HF self-care that could be personalised to adapt to symptom severity, age and cognitive status of patients. This could provide evidence-based reminders or questions to better ensure that all domains of HF self-care are addressed systematically over time. Given that social media can include functions that link users together, there is also scope for patients and caregivers to share successful strategies to better integrate HF selfcare behaviours with 'normal' life patterns, decrease isolation and increase social support.

\section{Limitations}

As with all reviews, the conclusions were constrained by the quality of the studies included and influenced by their populations, settings and foci. One particular issue raised in the studies related to the significance of HF self-care factors not identified by study participants in the findings reported. It is unclear whether, and to what degree, these factors were pursued during data collection. Most of the studies were carried out in wealthy countries with good access to effective HF medicines and health services. More research needs to be undertaken of self-care of HF in low and middle income countries and different health systems. There was also a lack of age-based analysis in the included studies and under-reporting of patients' New York Heart Association (NYHA) functional class; yet, these can reasonably be seen to influence capacity and skills in managing HF self-care.

Acknowledgements The Canadian Institutes of Health Research funded this review via a Knowledge Synthesis Grant (RESO 014 331). It was performed in conjunction with and indexed by the Joanna Briggs Institute as a systematic review (8:24 Suppl 2010).

Contributors AMC Conceived the review, coordinated the search and reviews, led the analysis and writing of manuscript drafts and acts as guarantor. MS, KH, JS, PHS, DRT and KC: Reviewed studies, and contributed to analysis and drafting of manuscript.

Funding Funding was provided via a CIHR Knowledge Synthesis Grant.

Competing interests None.

Provenance and peer review Not commissioned; externally peer reviewed.

\section{REFERENCES}

1 Lainscak M, Blue L, Clark AL, et al. Self-care management of heart failure: practical recommendations from the Patient Care Committee of the Heart Failure Association of the European Society of Cardiology. Eur J Heart Fail 2010;13:115-26.

2 Riegel B, Moser D, Anker SD, et al. State of Science: Promoting self care in persons with heart failure: A Scientific Statement from the American Heart Association. Circulation 2009;120:1141-63.

3 World Health Organisation. Adherence to long-term therapies: evidence for action. Geneva: WHO, 2003.

4 Heart Failure Society of America. HFSA 2006 Comprehensive Heart Failure Practice Guideline. St Paul, Minnesota: HFSA, 2006.

5 Heart Failure Society of America. Self care: following your treatment plan and dealing with your symptoms. St Paul, MN: Heart Failure Society of America, 2006

6 Chaudhry SI, Mattera JA, Curtis JP, et al. Telemonitoring in patients with heart failure. N Engl J Med 2010;363:2301-09.

7 Jaarsma $T$, van der Wal M, Lesman-Leegte I, et al. Effect of moderate or intensive disease management program on outcome in patients With heart failure coordinating study evaluating outcomes of advising and counseling in heart failure (COACH). Arch Intern Med 2008;168:316-24.

8 Nucifora G, Albanese M, De Biaggio P, et al. Lack of improvement of clinical outcomes by a low-cost, hospital-based heart failure management programme. J Cardiovasc Med 2006;7:614-22.

9 Smith B, Forkner E, Zaslow B, et al. Disease management produces limited quality-of-life improvements in patients with congestive heart failure: evidence from a randomized trial in community-dwelling patients. Am J Manag Care 2005; 11:701-3

10 Nguyen $V$, Ducharme $A$, White $M$, et al. Lack of long-term benefits of a 6-month heart failure disease management program. J Card Fail 2006;13:287-93.

11 Holland R, Brooksby I, Lenaghan E, et al. Effectiveness of visits from community pharmacists for patients with heart failure: HeartMed randomised controlled trial. BMJ 2007:334:1098.

12 Ledwidge $M$, Ryan E, O'Loughlin C, et al. Heart failure care in a hospital unit: a comparison of standard 3-month and extended 6-month programs. Eur J Heart Fail 2005:16:385-91.

13 Anker SDK, Koehler F, Abraham WT. Telemedicine and remote management of patients with heart failure. Lancet 2011(378):731-9.

14 McCall N, Cromwell J. Results of the Medicare health support disease-management pilot program. N Engl J Med 2011;365:1704-12.

15 Savard LA, Thompson DR, Clark AM. A meta-review of evidence on heart failure disease management programs: the challenges of describing and synthesizing evidence on complex interventions. Trials 2011;12:194.

16 Clark AM, Thompson DR. Heart failure disease management programmes: a new paradigm for research. Heart 2012;98:1476-7.

17 Clark AM, Thompson DR. The future of heart failure disease management programs. Lancet 2008;372:784-6.

18 Craig P, Dieppe $P$, Macltyre $S$, et al. Developing and evaluating complex interventions: the new Medical Research Council Guidance. BMJ 2008;337:979-83.

19 Clark AM, Reid ME, Morrison CE, et al. The complex nature of informal care in home-based heart failure management. J Adv Nurs 2008;61:373-83.

20 Molloy GJ, Johnston DW, Witham MD. Family caregiving and congestive heart failure. Review and analysis. Eur J Heart Fail 2005;7:592-603.

21 Pound $\mathrm{P}$, Britten N, Morgan $\mathrm{M}$, et al. Resisting medicines: a synthesis of qualitative studies of medication taking. Soc Sci Med 2005;61:133-55.

22 Campbell RP, Pope C, Britten N, et al. Evaluating meta-ethnography: a synthesis of qualitative research on lay experiences of diabetes and diabetes care. Soc Sci Med 2003:56:671-84

23 Jaarsmaa T, Strömbergb A, Mårtensson J, et al. Development and testing of the European heart failure self-care behaviour scale. Eur J Heart Fail 2003:5:363-70.

24 Noblit G, Hare R. Meta-ethnography: synthesizing qualitative data. London: Sage, 1988.

25 Clark A, King-Shier K, Thompson D, et al. A qualitative systematic review of influences on attendance at cardiac rehabilitation programs after referral. Am Heart J 2012;16:835-45.

26 Clark AM, Savard LA, Spaling MA, et al. Understanding help-seeking decisions in people with heart failure: a qualitative systematic review. Int J Nurs Stud 2012;49:1582-97.

27 NHS Learning and Development. Critical Appraisal Skills Programme (CASP) and evidence-based practice: CASP qualitative appraisal tool, 2007.

28 Granger BB, Sandelowski M, Tahshjain $\mathrm{H}$, et al. A qualitative descriptive study of the work of adherence to a chronic heart failure regimen: patient and physician perspectives. J Cardiovasc Nurs 2009;24:308-15

29 Dickson VV, McCauley LA, Riegel B. Work-heart balance: the influence of biobehavioral variables on self-care among employees with heart failure. AAOHN J 2008; 56:63-73.

30 Sloan RS, Pressler SJ. Cognitive deficits in heart failure: re-cognition of vulnerability as a strange new world. J Cardiovasc Nurs 2009;24:241-8.

31 Meyerson KL, Kline KS. Qualitative analysis of a mutual goal-setting intervention in participants with heart failure. Heart Lung 2009;38:1-9. 
32 Macabasco-OConnell A, Crawford MH, Stotts N, et al. Self-care behaviors in indigent patients with heart failure. J Cardiovasc Nurs 2008;23:223-30.

33 Rerkluenrit J, Panpakdee 0, Malathum $\mathrm{P}$, et al. Self-care among Thai people with heart failure. Thai J Nurs Res 2009;13:43-53.

34 Mead H, Andres E, Ramos C, et al. Barriers to effective self-management in cardiac patients: the patient's experience. Patient Educ Couns 2010;79:69-76.

35 Costello JA, Boblin S. What is the experience of men and women with congestive heart failure? Can J Cardiovasc Nurs 2004;14:9-20.

36 Riegel B, Dickson VV, Goldberg LR, et al. Factors associated with the development of expertise in heart failure self-care. Nurs Res 2007:56:235-43.

37 Clark AM, Freydberg N, McAlister FA, et al. Patient and informal caregivers' knowledge of heart failure: necessary but insufficient for effective self care. Eur J Heart Fail 2009;11:617-21.

38 Horowitz CR, Rein SB, Leventhal H. A story of maladies, misconceptions and mishaps: effective management of heart failure. Soc Sci Med 2004;58:631-43.

39 Jurgens $\mathrm{CY}$, Hoke L, Byrnes J, et al. Why do elders delay responding to heart failure symptoms? Nurs Res 2009;58:274-82.

40 Dickson V, CS L, Riegel B. How do cognitive function and knowledge affect heart failure self-care? J Mix Methods Res 2011:5:167-89.

41 Seto E, Leonard KJ, Cafazzo JA, et al. Self-care and quality of life of heart failure patients at a multidisciplinary heart function clinic. J Cardiovasc Nurs 2011;26:377-85

42 Mahoney JS. The illness experiences of patients and their family members living with congestive heart failure. University of Texas Health Science Center at Houston School of Nursing, 2000

43 Stull DE, Starling R, Haas G, et al. Becoming a patient with heart failure. Heart Lung 1999:28:284-92.

44 van der Wal MHL, Jaarsma T, Moser DK, et al. Qualitative examination of compliance in heart failure patients in The Netherlands. Heart Lung 2010:39:121-30.

45 Scotto CJ. The lived experience of adherence for patients with heart failure. J Cardiopulm Rehabil 2005:25:158-63.

46 Riegel B, Dickson VV, Cameron J, et al. Symptom recognition in elders with heart failure. J Nurs Scholarsh 2010;42:92-100.

47 Lough MA. Ongoing work of older adults at home after hospitalisation. J Adv Nurs 1996:23:804-09.

48 Jowsey $\mathrm{T}$, Jeon $\mathrm{Y}-\mathrm{H}$, Dugdale $\mathrm{P}$, et al. Challenges for co-morbid chronic illness care and policy in Australia: a qualitative study. Aust New Zealand Health Policy 2009;6:22.

49 Dickson VV, Deatrick JA, Riegel B. A typology of heart failure self-care management in non-elders. Eur J Cardiovasc Nurs 2008;7:171-81.

50 Buetow S, Goodyear-Smith F, Coster G. Coping strategies in the self-management of chronic heart failure. Fam Pract 2001;18:117-22.

51 Kaholokula JK, Saito E, Mau MK, et al. Pacific Islanders' perspectives on heart failure management. Patient Educ Couns 2008:70:281-91.

52 Scotto CJ. Factors influencing adherence to a prescribed health regimen for individuals with heart failure. Duquesne University School of Nursing, 2003.

53 Gary R. Self-care practices in women with diastolic heart failure. Heart Lung 2006;35:9-19.

54 Riegel B, Carlson B. Facilitators and barriers to heart failure self-care. Patient Educ Couns 2002;46:287-95.

55 Riegel B, Dickson VV, Hoke $L$, et al. A motivational counseling approach to improving heart failure self-care: mechanisms of effectiveness. J Cardiovasc Nurs 2006:21:232-41.

56 Riegel B, Dickson VV, Kuhn L, et al. Gender-specific barriers and facilitators to heart failure self-care: a mixed methods study. Int J Nurs Stud 2010;47:888-95.

57 Boren DM. Women's experiences with chronic heart failure. University of San Diego, 2001

58 Zambroski CH. Qualitative analysis of living with heart failure. Heart Lung 2003;32:32-40.
59 Weierbach FM. A description of community resource use by rural elders with heart failure. University of Virginia, 2008.

60 Wu J. Medication adherence in patients with heart failure. University of Kentucky, 2007.

61 Thornton N, Hopp FP. 'So I Just Took Over': African American daughters caregiving for parents with heart failure. Fam Soc J Contemp Soc Serv 2011;92:211-17.

62 Dickson V, McCarthy MM, Howe A, et al. Sociocultural influences on heart failure self-care among an ethnic minority black popoulation. J Cardiovasc Nurs 2013;28:111-18

63 Sanford J, Townsend-Rocchicciolli J, Horigan A, et al. A process of decision making by caregivers of family members with heart failure. Res Theory Nurs Pract 2011;25:55-70.

64 Scott LD. The effect of inotropic infusions on health-related quality of life for heart failure patients and their caregivers. University of Michigan, 1999.

65 Ming L, Hassali MA, Shafie AA, et al. Perspectives of heart failure patients in Malaysia towards medications and disease state management: Findings form a qualitative study. J Public Health 2011;19:569-77.

66 Schnell KN, Naimark BJ, McClement SE. Influential factors for self-care in ambulatory care heart failure patients: a qualitative perspective. Can J Cardiovasc Nurs 2006;16:13-19.

67 Reid M, Clark A, Murdoch DL, et al. Patients strategies for managing medication for chronic heart failure. Int J Cardiol 2006;109:66-73.

68 Wu JR, Moser DK, Lennie TA, et al. Factors influencing medication adherence in patients with heart failure. Heart Lung 2008;37:8-16.

69 Glassman KS. Older persons' experience of managing medication: the myth of compliance. New York University, 2007.

70 Falk S, Wahn AK, Lidell E. Keeping the maintenance of daily life in spite of Chronic Heart Failure. A qualitative study. Eur J Cardiovasc Nurs 2007;6:192-99.

71 Bennett SJ, Cordes DK, Westmoreland G, et al. Self-care strategies for symptom management in patients with chronic heart failure. Nurs Res 2000:49:139-45.

72 Clark AM, McMurray JJV, Morrison CE, et al. A qualitative study of the contribution of pharmacists to heart failure management in Scotland. Pharm World Sci 2005;27:453-8.

73 Hoyt CA. Navigating to safe harbor: Problems and processes of living with heart failure. University of Kentucky, 1999

74 Stromberg A, Brostrom A, Dahlstrom U, et al. Factors influencing patient compliance with therapeutic regimens in chronic heart failure: a critical incident technique analysis. Heart Lung 1999;28:334-41.

75 Helleso R, Eines J, Fagermoen M. The significance of informal caregivers in information management from the perspective of heart failure patients. I Clin Nurs 2012:21:495-503.

76 Dickson VV, Tkacs N, Riegel B. Cognitive influences on self-care decision making in persons with heart failure. Am Heart J 2008a;154:424-31.

77 Rutledge $T$, Reis $V$, Linke $S$, et al. Depression in heart failure: a meta-analytic review of prevalence, intervention effects, and associations with clinical outcomes. J Am Coll Cardiol 2006; $48: 1527-37$

78 Cameron J, Ski CF, McLennan SN, et al. Development of the Heart Failure Screening Tool (Heart-FaST) to measure barriers that impede engagement in self-care. Eur J Cardiovasc Nurs 2013. [Epub ahead of print 22 Aug 2013] doi:10.1177/ 1474515113502461

79 Gallacher K, May C, Montori VM, et al. Understanding patients' experiences of treatment burden in chronic heart failure using normalization process theory. Ann Fam Med 2011;9:235-43.

80 Aubin M, Giguere A, Martin M, et al. Interventions to improve continuity of care in the follow-up of patients with cancer. Cochrane Database Syst Rev 2012;8: CD007672.

81 O'Connor AM, Bennett CL, Stacey D, et al. Decision aids for people facing health treatment or screening decisions. Cochrane Database Syst Rev 2009;3:CD001431.

82 Black AD, Car J, Pagliari $C$, et al. The impact of eHealth on the quality and safety of health care: a systematic overview. PLOS Med 2011;8:e1000387. 